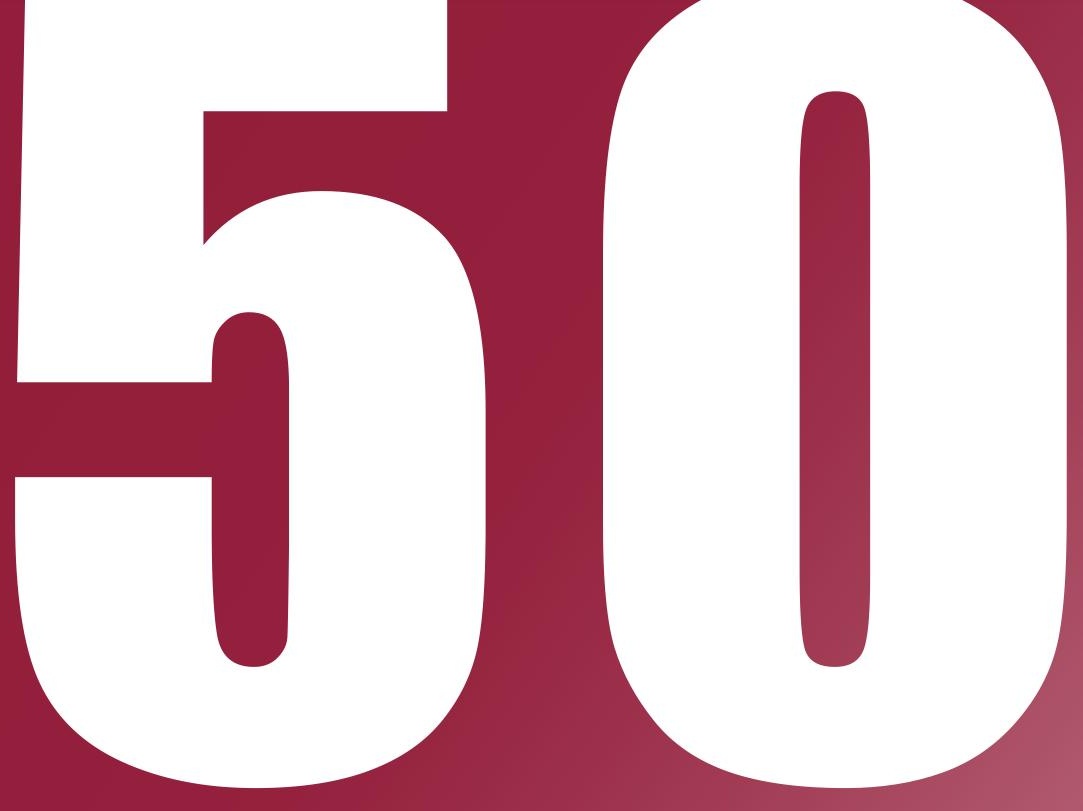

ÁMBITOS

REVISTA

INTERNACIONAL

DE

COMUNICACIÓN

$\mathbf{N}^{\circ} \mathbf{5 0}$

EDICIÓN OTOÑO

2020

ISSN: 1139-1979

E-ISSN: 1988-5733

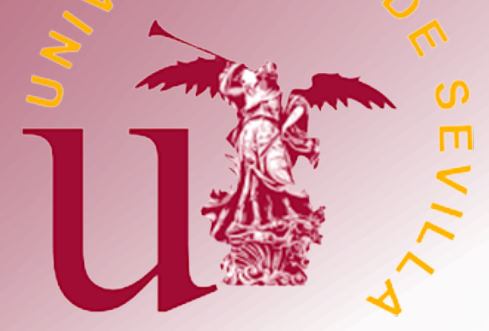




\section{INDICE}

EDITORIAL EDITORIAL

Presentación del Monográfico. Comunicación y juventud. Nuevos medios, representación, recepción y participación en contenidos de entretenimiento e información

Presentation of the monograph. Communication and youth. New media, representation, reception and participation in entertainment and information content.

Juan Francisco Gutiérrez Lozano, Francisco Javier Ruiz del Olmo

7-9

\section{MONOGRÁFICO MONOGRAPH}

El consumo audiovisual de la Generación Z. El predominio del vídeo online sobre la televisión tradicional The audiovisual consumption of Generation $Z$. The predominance of online video over traditional television

La imagen de los jóvenes en las estrategias transmedia de las series de televisión: el caso de Skam España en Instagram

The image of young people in transmedia strategies of TV series: the case of Skam España on Instagram

Sergio Jesús Villén Higueras, Francisco Javier Ruiz del Olmo

La penetración de Netflix en el público español ¿cuestiona el modelo televisivo tradicional?

Does the arrival of Netflix in the Spanish audience challenge the traditional television model?

Javier Bustos Díaz

49-61

Generación Z y consumo de información política: entre la televisión y los nuevos formatos mediáticos Generation $Z$ and consumption of political information: between television and new media formats

Ruth Gómez de Travesedo Rojas, Marta Gil Ramírez

$62-79$

Actualidad y comedia: El éxito de El Intermedio y La Resistencia entre los espectadores jóvenes

Information and comedy: The Success of El Intermedio and La Resistencia for young audiences

Inmaculada Concepción Aguilera García

80-95 
El efecto socializador de articular un espacio de comunidad virtual en el ecosistema del aula

The socializing effect of creating a virtual community space in the classroom environment

Lorea Ariadna Ruiz Gómez, Mónica Hinojosa Becerra, Francisco Javier Ruiz San Miguel

Juventude ciberativista e educação: reflexões sobre um jeito hacker de ser

Cyber activity youth and Education: reflections on a hacker way of being

Carla Azevedo de Aragão, Pietro Matheus Bompet Fontoura Alves, Karina Moreira Menezes

La risa grotesca en la obra de Bob Dylan: análisis de la Bacanal y el Festín Carnavalesco en la trilogía folk-rock eléctrica

Grotesque Laughter in Bob Dylan's Work: Analysis of The Bacchanal and the Carnival Feast in the Electric Folk-rock Trilogy

Jesús Albarrán Ligero

El papel del intertexto en el videojuego. Una partida, mil y una historias

Intertext in video games. A game, thousand and one stories

Rocío Serna-Rodrigo

145-158

\section{ÁmBitos PERSONALES PERSONAL ÁmBITOS}

El auge de Twitch: nuevas ofertas audiovisuales y cambios del consumo televisivo entre la audiencia juvenil The rise of Twitch: New audiovisual offers and the transformation of Television consumption among young audiences

\section{ARTÍCULOS ARTICLES}

La recepción de los medios de comunicación para residentes rusos en la Costa del Sol

Mass media reception by Russian residents on the Costa del Sol

Natalia Meléndez Malavé, José Carlos Pozo García

176-192

La calidad periodística en la cobertura de terremotos: Caso Ecuador

Journalistic quality in earthquake coverage: The case of Ecuador

Juan Pablo Arrobo-Agila, Mendoza María, Ignacio Aguaded

La credibilidad publicitaria en la nueva esfera mediática: los universitarios y los medios

Advertising credibility in the new media sphere: university students and the media 
Arco iris en medios brasileños: percepciones sociales sobre una campaña publicitaria LGBTQIA+

Rainbow in Brazilian media: social perceptions of an LGBTQIA+ advertising campaign

Leonardo Ferreira Batista, José Geraldo de Araújo Ramalho Filho

224-239

Rituales y comunicación política: la toma de posesión de los presidentes autonómicos españoles

Rituals and political communication: the Spanish autonomous Presidents inauguration

Ricardo Domínguez García

240-258

RESEÑAS REVIEWS

Una mirada diferente para hacer periodismo

A different look for making journalism

Guillermo Paredes-Otero

259-261

Periodismo y tecnología: una simbiosis continua

Journalism and technology: a continuous symbiosis

Luisa Graciela Aramburú Moncada

262-265

Una propuesta metodológica para impartir la asignatura de redacción periodística

Journalism and technology: a continuous symbiosis

Noelia Ruiz-Alba

266-268 


\title{
Arco iris en medios brasileños: percepciones sociales sobre una campaña publicitaria LGBTQIA+
}

\author{
Rainbow in Brazilian media: social perceptions of an LGBTQIA+ advertising \\ campaign
}

\author{
Leonardo Ferreira Batista \\ Universidad Federal de Campina Grande (PPGA) Aprígio Veloso, 882 - Bairro Universitário - \\ Campina Grande, Paraíba 58428-830 | Brasil| https://orcid.org/0000-0002-7388-1297 \\ |leonardofb.leo@gmail.com

\section{José Geraldo de Araújo Ramalho Filho} \\ Centro de Educación Superior Reinaldo Ramos (CESREI) | Fernandes Vieira, \\ 1210 - Mirante, Campina Grande, Paraíba 58407-490 | Brasil| http://orcid.org/0000-0002- \\ 4722-2423 | geraldoaramalhof@gmail.com
}

Fechas | Recepción: 20/05/2020 | Aceptación: 03/08/2020 | Publicación final: 15/10/2020

\begin{abstract}
Resumen
Este artículo analiza las percepciones de los representantes del movimiento LGBTQIA+ (lesbiana, gay, bisexual, transexual, queer, intersexual, asexual/género y más) sobre la campaña para la edición especial de la marca Doritos Rainbow, con el Objetivo de discutir cómo algunos miembros del movimiento LGBTQIA+ ven la visibilidad de la causa social que es la esencia de esta comunidad y los avances que puede lograr mediante la inserción en los medios. El estudio muestra relevancia teórica para abordar un tema social prominente que está entrando cada vez más en el espacio publicitario y necesita investigación. A partir de la revisión de la literatura y los procedimientos de análisis del discurso, se discutió la necesidad de producir más
\end{abstract}

\begin{abstract}
This article analyzes the perceptions of representatives of the LGBTQIA+ movement (lesbian, gay, bisexual, transsexual, queer, intersex, asexual/gender and more) about the campaign for the special edition of the Doritos Rainbow brand, with the aim of discussing how some members of the LGBTQIA+ movement see the visibility of the social cause that is the essence of this community and the progress it can achieve through insertion in the media. The study shows theoretical relevance to address a prominent social issue that is increasingly entering the advertising space and needs research. Based on the literature review and discourse analysis procedures, the need to produce more advertising campaigns with a social focus and
\end{abstract}


campañas publicitarias con un enfoque social y cómo se pueden configurar estas campañas para lograr el resultado deseado. En este sentido, la percepción principal de los sujetos entrevistados fue que, a pesar de que benefician la visibilidad del movimiento en la sociedad, estas campañas deberían estar más alineadas con la historia y la filosofía popular de este movimiento, así como sus composiciones más alineadas con el público objetivo. Por lo tanto, los resultados mostraron que, además de acercar al público al producto, es necesario tener un conocimiento real de la causa como un punto destacado en las piezas publicitarias para que los temas de la campaña se vean realmente afectados por el mensaje de atractivo social transmitido.

Palabras clave: publicidad, medios brasileños, movimiento LGBTQIA+, Doritos Rainbow, causa social. how these campaigns can be configured to achieve the desired result were discussed. In this sense, the main perception of the subjects interviewed was that, although they benefit the visibility of the movement in society, these campaigns should be more aligned with the history and popular philosophy of this movement, as well as its more aligned compositions. with the target audience. Therefore, the results showed that, in addition to bringing the public closer to the product, it is necessary to have a real knowledge of the cause as a prominent point in the advertising pieces so that the campaign themes are really affected by the attractive message social broadcast.

Keywords: advertising, brazilian media, LGBTQIA+ movement, Doritos Rainbow, social cause.

\section{INTRODUCCIÓN}

El movimiento social titulado LGBTQIA+ Movement, un acrónimo que representa el grupo formado por lesbianas, gais, bisexuales, transexuales, queers, intersexuales, asexuales / géneros y más, está cada vez más presente en la publicidad, de tal manera que su visibilidad ha ganado diferentes perspectivas. Esto sugiere la necesidad de investigar si las producciones publicitarias con este enfoque pueden transmitir información real que está histórica y socialmente alineada con la causa (Kotler, Kartajaya, y Setiawan, 2019).

Desde la aparición del movimiento, ahora entendido como LGBTQIA+, la diversidad sexual y de género ha ganado terreno y ha sido objeto de muchas discusiones. De tal manera que el papel de la publicidad en la difusión de estas reflexiones es significativo en la reasignación del conocimiento sobre la lucha histórica de los "LGBTQIA+'s", un nombre utilizado para la reunión de miembros del movimiento, lo que refuerza la necesidad de exposición para alcanzar cambio social (Carvalho y Lisboa Filho, 2019; Rodrigues y Carvalho, 2015).

La publicidad funciona con ideas, imágenes, música y otros que pueden aportar una forma alternativa de conciencia, e incluso educativa, en su ámbito de acción. En este campo, el concepto de identidad puede reforzarse mediante el reconocimiento de los grupos sociales (Carvalho y Lisboa Filho, 2019; Santos y Cunha, 2019), aportando el efecto de compra puro que busca el mercado o el altruismo frente a la causa evidenciada (Carvalho y Lisboa Filho, 2019; Rodrigues y Carvalho, 2015).

Y esto se acentúa cuando implica la inserción de la causa social (Kotler et al., 2019), un fenómeno recurrente en los medios que ha transformado la forma de hacer comunicación y ciencia. De tal 
manera que se justifica en la necesidad del desarrollo de la humanidad y en la contribución teórica en línea con la dinámica de las relaciones sociales y la dinámica de sus nuevos reordenamientos. Para investigar las percepciones del público más interesado, los LGBTQIA+, este artículo busca responder la siguiente pregunta de investigación: ¿Cuáles son las percepciones del público LGBTQIA+ sobre la visibilidad del movimiento al que pertenecen en la publicidad brasileña, especialmente en la campaña de la marca Doritos Rainbow del año 2019?

El objetivo de este estudio es comprender, bajo las percepciones de un grupo de sujetos pertenecientes al Movimiento LGBTQIA+, cuál es la alineación de la pieza publicitaria analizada con la causa social en cuestión. Basado en la literatura sobre inclusión social en publicidad (Kotler et al., 2019), se pretende identificar qué hipótesis se confirma: a) la visibilidad del Movimiento LGBTQIA+ en la pieza publicitaria analizada promueve la alineación con la historia popular y la filosofía para la inclusión fenómeno social; b) a pesar de la presencia de la visibilidad del Movimiento LGBTQIA+ en la campaña publicitaria analizada, no existe una alineación con la historia y la filosofía popular para la inclusión social del fenómeno; o c) en la pieza publicitaria no hay representatividad o alineación con la historia popular y la filosofía del movimiento que promueve la inclusión social del fenómeno. Además, para enriquecer esta discusión, se consideran posibles peculiaridades en los discursos de los sujetos.

Para esto, se construyó una investigación cualitativa a partir de la revisión de la literatura, para la discusión teórica, el análisis del objeto de estudio, la campaña «Nada es más bold que ser tú mismo» de la edición especial de la marca Doritos Rainbow y la percepción de los problemas involucrados en la causa, que luego incluyó el análisis del discurso de los sujetos involucrados en el tema. Por lo tanto, el estudio se defiende como significativo para los avances sociales basados en estudios de comunicación de medios (Kotler et al., 2019).

El estudio de la campaña elegida destaca un tema que aún está surgiendo en la ciencia, la causa LGBTQIA+, promueve una importante reflexión social y contribuye gerencialmente a la práctica de la publicidad basada en su contribución a la sociedad (Kotler et al., 2019). Este tipo de investigación es importante para el avance de los estudios sociales y de marketing.

Además de esta introducción, este estudio está estructurado en la aclaración científica teórica sobre los temas discutidos, los procedimientos metodológicos seguidos, los resultados obtenidos y las discusiones que permiten una comprensión más profunda de la investigación y el consecuente logro del objetivo previsto.

\section{EL MOVIMIENTO LGBTQIA+ EN BRASIL}

La lucha social de los LGBTQIA+ ha ido ganando más fuerza con las movilizaciones y el alcance popular de los movimientos sociales presentes en la sociedad brasileña, principalmente en términos mediáticos. Sin embargo, todavía hay muchas barreras culturales, sociales e incluso morales que enfrentar. Una solución a esto puede ser el intento de hacer que el movimiento sea visible como un movimiento sólido, adecuado y ampliamente necesario.

Este movimiento surgió en 1978, como lo propugna la sociología política, a partir de la 
formalización del movimiento LGBTQIA+ brasileño, a través de varios grupos activistas de la causa, como el Movimiento Homosexual Brasileño (MHB) (Bortolozzi, 2019; Ferreira y Sacramento, 2019). Luego, en el período de la dictadura militar, la confrontación de una represión que, además del movimiento LGBTQIA+, combatía con movimientos de identidad, como el movimiento negro y el movimiento feminista (Ferreira y Sacramento, 2019).

En 2018, el Movimiento LGBTQIA+ (como se lo llamó) completó 40 años de actividad en Brasil, que comenzó con Somos -grupo de afirmación homosexual- en 1978. Productos culturales como libros, revistas, documentales y periódicos con un enfoque hacia estos temas se lanzaron (Ferreira y Sacramento, 2019) y se convirtieron en instrumentos políticos importantes (Bortolozzi, 2019) en busca de la visibilidad como un camino hacia la ciudadanía, reflejado en los derechos políticos, sociales y civiles (Ferreira y Sacramento, 2019), que tienen visibilidad en los medios y alcance popular como precursores (Facchini, 2009). Dada esta perspectiva, el movimiento LGBTQIA+ creció en número de grupos y expandió su visibilidad, su red social y sus espacios (Facchini, 2009).

El acrónimo LGBTQIA+ está en constante construcción, como resultado de identidades afirmativas. La atención se centra en cuestiones afectivas y de género, una centrada en el campo sexual y la otra relacionada con el reconocimiento del cuerpo individual. Esto se logró con la siguiente evolución cronológica:

Tabla 1

Nomenclaturas del movimiento LGBTQA+ en el pasado

\begin{tabular}{|c|c|}
\hline Nomenclatura & Período \\
\hline MHB (Movimiento Homosexual Brasileño) & Hasta 1993 \\
\hline Movimiento GLT (gais, lesbianas y travestis) & Desde 1995 \\
\hline $\begin{array}{c}\text { Movimiento GLBT (gay, lesbiana, bisexual y transgénero) con las variantes GLTB y LGBT } \\
\text { Movimiento LGBTIA+ (lesbiana, gay, bisexual, transgénero, homosexual, intersexual, } \\
\text { asexual/género y más) }\end{array}$ & Desde 2018 \\
\hline
\end{tabular}

Fuente: elaboración propia.

La transferencia de la letra "L" al principio sería la necesidad y el intento de aumentar la visibilidad de las lesbianas en el contexto (Facchini, 2009; Facchini y Rodrigues, 2018; Rodrigues y Carvalho, 2015). Hoy hablamos de representaciones de lesbianas, gais, bisexuales, transexuales, homosexuales, intersexuales, asexuales / de género y más (LGBTQIA+) como una forma de representar cambios en la interpretación de los roles sociales, tan necesarios para la evolución de la sociedad. Del mismo modo, las configuraciones familiares, previamente reguladas por valores morales y biológicos (Carvalho y Lisboa Filho, 2019), hoy en día son ampliamente multiplicadas y multifacéticas.

Aunque presente en las mentes de los consumidores que pertenecen a este movimiento, la causa social discutida aún necesita perspectivas teóricas para avanzar y generar reflexiones, 
incluido el hecho de que se relaciona con teorías sustanciales de inclusión social en la publicidad (Kotler, Kartajaya, y Setiawan, 2010; Kotler y KELLER, 2012; Rodrigues, 2018), por lo que existe un verdadero propósito de contribuir a la sociedad.

Por lo tanto, es necesario que haya un enfoque social en el entorno comunicacional para causas como esta, que están presentes y cada vez más vivas dentro de la sociedad brasileña, y que no son diferentes en todas partes del mundo, como lo recomiendan estudios en diferentes áreas del conocimiento en la intersección con el tema (Bortolozzi, 2019; Bragaglia, 2019; Carvalho y Lisboa Filho, 2019; Facchini, 2009; Ferreira y Sacramento, 2019; Peralta, Martins y Santana, 2019; Rodrigues, 2018; Santos y Cunha, 2019).

\subsection{LGBTQIA+ Visibilidad en medios brasileños}

El enfoque en el comportamiento del consumidor enfatiza y moviliza las acciones de los medios para el estudio de compras, usos y disposiciones de bienes, servicios, ideas y/o experiencias dirigidas a satisfacer necesidades y deseos (Kotler y Keller, 2012). Sin embargo, el aspecto social ha sido un instrumento de mayor proximidad a los consumidores en las discusiones sobre el desarrollo de la publicidad y su alineación con las causas sociales (Kotler et al., 2019).

En esta perspectiva, el discurso publicitario se vuelve ambiguo, ya que involucra el sesgo de mercado comúnmente intrínseco y la representación antropológica presente en el ámbito social (Gastaldo, 2013). Entonces, comprender la visibilidad que tiene un movimiento social en los medios y sus implicaciones y reacciones sociales es un paso significativo hacia la comprensión de la contribución de la comunicación de los medios al desarrollo de la sociedad, así como el monitoreo real y sólido de los fenómenos que a menudo surgen de las transformaciones sociales.

Desde su aparición, el movimiento LGBTQIA+ ha avanzado mucho en el reconocimiento de la diversidad sexual y de género. Sin embargo, la mayoría de las producciones publicitarias benefician predominantemente al mercado, debido a tendencias sesgadas en el enfoque en la venta de productos finales (Kotler et al., 2019).

En contraste, el papel de estas producciones es significativo al resignificar las representaciones realizadas y necesita alinearse con la lucha histórica del movimiento en cuestión, brindando visibilidad real e inserción social (Carvalho y Lisboa Filho, 2019; Kotler et al., 2019; Rodrigues y Carvalho, 2015).

La representación busca referencias en ideas, imágenes, música, entre otros signos difundidos por los medios de comunicación, reforzando el carácter social, étnico, sexual y/o de clase. Por lo tanto, el concepto de representación puede desencadenar el de identidad, lo que se traduce en el reconocimiento de grupos sociales (Carvalho y Lisboa Filho, 2019). Con esto, se genera la búsqueda del efecto de compra, en el que el discurso publicitario ya no intenta simplemente persuadir, sino que también intenta generar un vínculo de identificación entre la causa y el público (Santos y Cunha, 2019). 
Desde esta perspectiva, uno puede entender la publicidad emocional, que, desde la década de 1990, busca crear piezas con cierto atractivo emocional, en las que el protagonista es más que el cliente, es una causa social, que genera identificación y compromiso de las minorías sociales (Bragaglia, 2019; Kotler et al., 2019). Herramienta importante en el proceso de conquista del público a través de la comunicación dotada de marcas de afinidad con los deseos del público objetivo.

No obstante, es necesario comprender que el consumidor LGBTQIA+ (también parte de la causa) exige mucho del mercado. El conocimiento histórico, la segmentación de la audiencia y las estrategias siempre deben estar presentes desde el punto de vista del mercado (Kotler et al., 2019; Peralta et al., 2019), ya que dos frentes promueven grandes desafíos, la verdadera representatividad de la causa y la conquista de consumidores críticos y más exigentes por la calidad de los productos y servicios para ellos mismos.

Por lo tanto, para esta audiencia, involucrada en temas sociales más complejos y controvertidos, se necesita más atención y responsabilidad cuando se realizan campañas publicitarias, que siguen la lógica de la inserción de causas sociales en la publicidad (Kotler et al., 2019; Peralta et al., 2019).

Hay visibilidad en las formas políticas y sociales y la lógica del mercado llamada gay friendly, es decir, "simpatizante" del movimiento LGBTQIA +. La publicidad entra en una arena conceptual e ideológica, tratando de ser fiel a lo que la causa exige y poniéndose en una disputa de percepciones y juicios ante la sociedad. Esta práctica, a veces ventajosa, a veces peligrosa, a veces controvertida, tiene un gran potencial de viralización en un contexto de consumidoresproductores de contenido e incluso defensores de marcas activas (Kotler et al., 2019; Rodrigues, 2018).

De esta manera, el enfoque se centra en el conocimiento, en comprender históricamente y verificar si las campañas producidas consideran no solo la pieza publicitaria en la agenda, sino características históricas y representativas en la planificación y ejecución de estas campañas, para hacer que la publicidad sea confiable y saludable para estos movimientos. Y la falta de comprensión consolidada de la contribución real de la visibilidad de esta causa en los medios de comunicación brasileños es el foco principal de las discusiones, lo que hace que la investigación sobre el tema sea tan significativa.

\section{PROCEDIMIENTOS METODOLÓGICOS}

Este artículo es el producto de una investigación cualitativa que analiza la campaña «Nada es más bold que ser tú mismo» de la edición especial de la marca Doritos Rainbow, creada por la agencia de publicidad AlmapBBDO en 2019, cuyo objetivo es analizar una variedad de percepciones de algunos miembros del movimiento LGBTQIA+ sobre la visibilidad y los avances de la causa social defendida por ellos a través de la inserción en los medios.

Busca aclarar en profundidad un fenómeno contemporáneo (Llewellyn y Northcott, 2007; Patton, 2014) para proporcionar una comprensión sobre la inmersión de la publicidad en las 
relaciones sociales y sus implicaciones en el contexto brasileño. Para esto, se realiza la descripción de la campaña de Doritos Rainbow (2019) y una discusión adicional al respecto.

A partir de este análisis, se comprende el avance del conocimiento del acceso del movimiento LGBTQIA+ a la publicidad. EI método utilizado permite la organización de datos sociales de tal manera que la realidad social se evidencia en detalle, confrontando varios datos (Duarte y Barros, 2006).

Para la recolección de datos, se utilizaron investigaciones documentales y entrevistas semiestructuradas, que proporcionaron información sobre el objeto de estudio, los sujetos y la observación empírica del conocimiento teórico. Los temas abordados contribuyen significativamente a aclarar el problema, en función de sus percepciones.

La campaña elegida se destaca por resaltar la causa LGBTQIA+, incitar los debates sociales a través de la reacción a los estímulos visuales e intentar incluir personajes del movimiento como una forma de llegar al público a través de la publicidad. Temas como la diversidad sexual están presentes en el eslogan, en la narrativa de la campaña y en la política interna de la empresa Pepsico, propietaria de la marca Doritos, que justifica la elección de este objeto como núcleo de la investigación.

Veintiún sujetos fueron entrevistados en persona, con siete discursos marcados por percepciones significativas elegidas para el análisis. Llamados S1, S2, S3, S4, S5, S6 y S7, son todos pertenecientes al movimiento LGBTQIA+ y viven en más de un lugar y sin criterios de edad. Por lo tanto, se justifica que las percepciones se eligieron a discreción de los investigadores y se centran en el objeto estudiado como una experiencia social del movimiento, sin tener el objetivo de dilucidar las estadísticas (Malhotra, 2011), sino más bien discutir subjetivamente la inserción social de un movimiento de identidad minoritario en los medios de comunicación brasileños a través de una campaña publicitaria (Kotler et al., 2019).

Los sujetos entrevistados contribuyeron al logro del objetivo de la investigación, sin un número inicialmente delimitado de participantes, ya que el método de saturación teórica argumenta que la repetición y la consecuente consolidación de la información obtenida justifican la ausencia de nuevas entrevistas (Minayo, 2017). El apoyo teórico, a su vez, se obtuvo de una revisión de la literatura con estudios relevantes y actualizados sobre los temas involucrados, la mayoría de los cuales fueron artículos indicados en el portal de revistas CAPES (Coordinación para la Mejora del Personal de Educación Superior), institución que, entre otras funciones, evalúa las revistas de impacto.

Los datos fueron analizados a través del análisis del discurso, ya que "las estructuras narrativas se convierten en discurso cuando el sujeto de la enunciación lo asume: hace una serie de elecciones, de persona, espacio, tiempo y figuras, contando la historia desde un cierto punto de vista" (Gregolin, 1995, p. 16, nuestra traducción).

Se eligió este tipo de análisis porque el discurso en sí es uno de los aspectos que materializa la ideología de los sujetos en función de sus sentimientos de pertenencia a la arena discursiva, dentro de un ámbito de formaciones imaginarias que relacionan mutuamente el tema y el 
emisor del discurso y los validan. como agentes satisfactorios para un análisis del conocimiento (Pêcheux, 1990), que busca confrontar sus percepciones con la publicidad representada por la pieza publicitaria en cuestión.

En el análisis, el investigador, ahora analista lingüista, entiende el lenguaje como una forma de intervención y articula la construcción de nuevos conocimientos a partir de la realidad, lo que requiere articulación con otras perspectivas, incluidas las interdisciplinarias (Rocha y Deusdará, 2005).

Este procedimiento garantiza una mayor neutralidad en la investigación, permitiendo la comprensión social de los discursos de los sujetos y transformando las percepciones en posibilidades reales de hacer ciencia (Rocha y Deusdará, 2005). Finalmente, concomitantemente, se alcanza el objetivo del estudio y la consiguiente respuesta al problema inicial.

\section{RESULTADOS Y DISCUSIONES}

\subsection{Análisis del discurso}

Este análisis se centró en discusiones que exploran el espacio de pertenencia de los sujetos relacionados con la inclusión de la causa social del Movimiento LGBTQIA+ en la publicidad brasileña, en la producción discursiva que surge de este corte de percepciones, en el conocimiento del mundo anterior sobre los temas más experimentados que cada uno de ellos tiene y sus contribuciones como sujetos y público de esta campaña, que ya ha sido creada con la ideología de participación democrática de esa comunidad.

Para preservar la identidad de los sujetos entrevistados en esta investigación, cada participante fue identificado por las siguientes indicaciones: S1, S2, S3, S4, S5, S6 y S7. Vale la pena mencionar que todos los participantes son parte de la comunidad analizada por esta investigación y fueron elegidos a discreción de la diversificación del perfil, lo que condujo a un mayor intercambio de información obtenida en sus discursos en función de sus experiencias de consumo y conocimiento de la historia y filosofía popular del movimiento LGBTQIA+. La Tabla 2 destaca los perfiles de los entrevistados.

Tabla 2

Perfiles de los entrevistados cuyos discursos fueron seleccionados para análisis

\begin{tabular}{|c|c|c|c|}
\hline Identificación & Género & Condición sexual & Años de edad \\
\hline S1 & hombre & gay & 30 \\
\hline S2 & hombre & queer & 25 \\
\hline S3 & hombre & gay & 40 \\
\hline S4 & mujer & lesbiana & 33 \\
\hline S5 & hombre & gay & 32 \\
\hline S6 & mujer & bisexual & 29 \\
\hline S7 & hombre & bisexual & 28 \\
\hline
\end{tabular}

Fuente: elaboración propia. 
Como construcción social, los sujetos consultados pertenecen al Movimiento LGBTQIA+, presentando una muestra no probabilística, elegida en la oportunidad y perteneciente a diferentes clases sociales y niveles de conocimiento (Malhotra, 2011). Por lo tanto, el criterio de accesibilidad y corte cualitativo de las percepciones subjetivas tiene su alcance apropiado para el análisis del discurso y sugiere parsimonia en la generalización de los resultados y las discusiones, proporcionando una comprensión que plantea futuras discusiones en la literatura.

\subsubsection{Consideraciones sobre el objeto analizado: la campaña Doritos Rainbow (2019)}

Para lograr el objetivo de este trabajo, la campaña publicitaria para la merienda Doritos se consideró como un objeto de estudio, en una versión especial dirigida al movimiento LGBTQIA+ brasileño más específicamente, ya que es una campaña significativamente conocida y presente en los medios y aborda explícitamente los componentes. que permiten un análisis enriquecido por parte de los sujetos, como videos, pancartas y discursos de personajes sobre temas relacionados con la causa.

Es importante entender que las marcas apuestan cada vez más por la inserción social discutida (Carvalho y Lisboa Filho, 2019; Rodrigues y Carvalho, 2015; Santos y Cunha, 2019). Esto se debe a que garantiza una buena imagen para las marcas y refleja un movimiento mediático que indirectamente los invita a todos a una postura socialmente correcta, responsable $y$ colaboradora.

Es el caso de Pepsico, una marca que fabrica el famoso producto Doritos, un tipo de sal que está a la venta en el mercado brasileño. En una versión con empaque de color, que hace referencia a la bandera del Movimiento LGBTQIA +, se coloca como un inductor de la visibilidad de la diversidad y las causas de su alcance, como se muestra en la Figura 1.

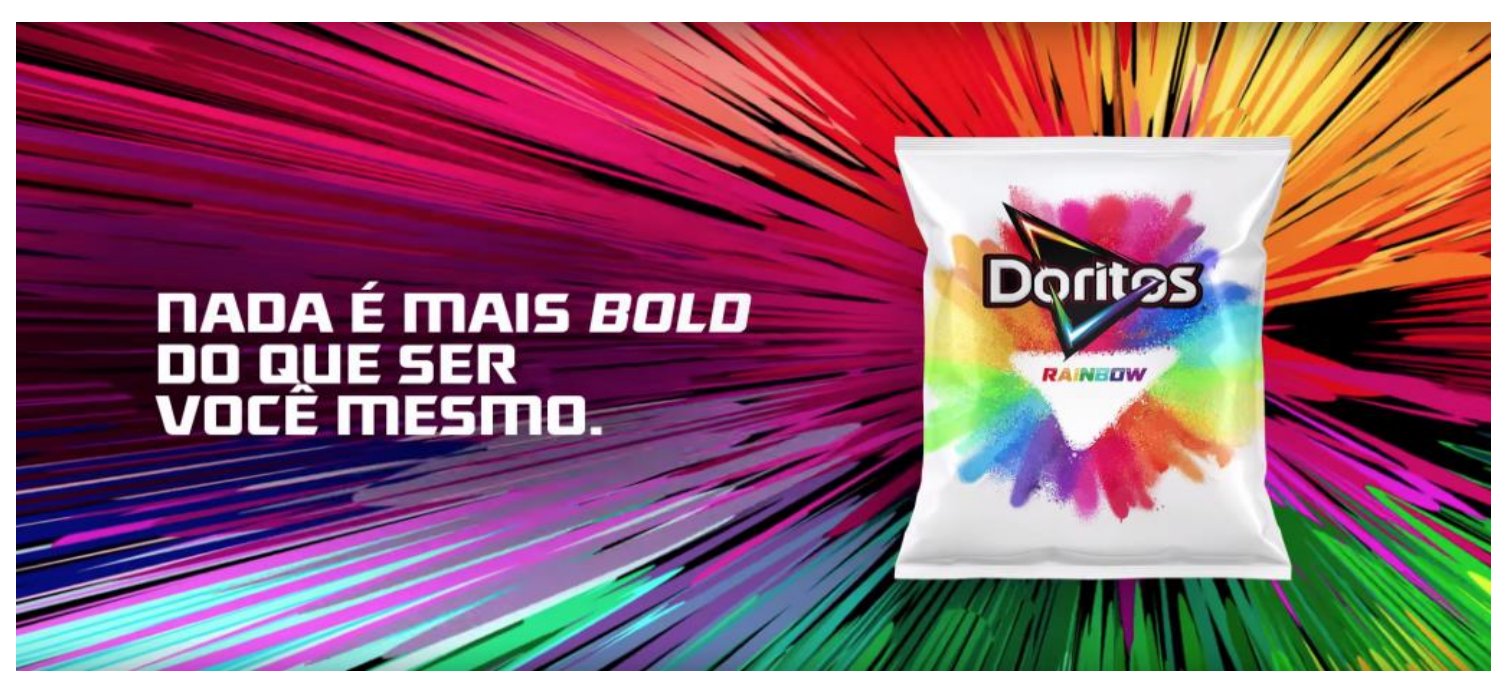

Figura 1. Banner de la campaña Doritos Rainbow

Fuente: sitio web AlmapBBDO, creador de la campaña (2019).

Esta versión del producto se llamaba Doritos Rainbow, debido a la referencia a un arco iris, un símbolo que se usa para describir la diversidad de miembros que pertenecen al grupo en estudio. 
La distribución y venta de este producto se realizó en Rock In Rio 2019, un evento que tiene lugar en la ciudad de Río de Janeiro y cuenta con una gran audiencia, en su mayoría jóvenes. En esta perspectiva, también tenían la propuesta de que la cantidad recaudada por la venta del producto se utilizaría para apoyar a las instituciones que promueven el desarrollo de la causa LGBTQIA+.

La campaña para publicitar este producto se tituló «Nada es más bold que ser tú mismo» como una forma de alentar la autenticidad y la libertad de estos actores sociales para ser felices con sus estilos de vida y decididos a vivir como ciudadanos. También se entiende que ser valiente y tener una voz socialmente activa es la forma más correcta de fortalecer el movimiento social en la agenda, generar una relación de identidad entre el eslogan y el público interesado, y aún tratar de asumir el papel de la conciencia social de una causa (Kotler et al., 2019).

El video comienza con Guigo (miembro del primer grupo de rap gay en Brasil), uno de los representantes, visto como una autoridad del habla en Brasil específicamente para este propósito, en defensa de frases que caracterizan la conciencia y la empatía para el público objetivo, como "Agregar fuerzas, buscar la paz". Los personajes se intercalan recitando más líneas de guía de la campaña. Drag queens, cantantes y artistas en general dan la nota clave a la campaña.

"Unir las diferencias entre usted y yo es lo que nos hace tan especiales" es la línea más emblemática del guion, porque resume el estímulo y la emancipación que la pieza publicitaria quiere causar cuando entra en contacto con el público, creando una especie de encanto y sentimiento de pertenencia a la causa. Murillo Zyess canta, inmediatamente después del extracto: "La diferencia es precisamente ser capaz de ser tú, no busques ningún patrón que encaje", lo que refuerza el carácter de identidad que hace del movimiento un auténtico defensor de su causa social. Otras frases siguen esta misma perspectiva y presentan los caracteres considerados representativos, como se muestra en la Figura 2.

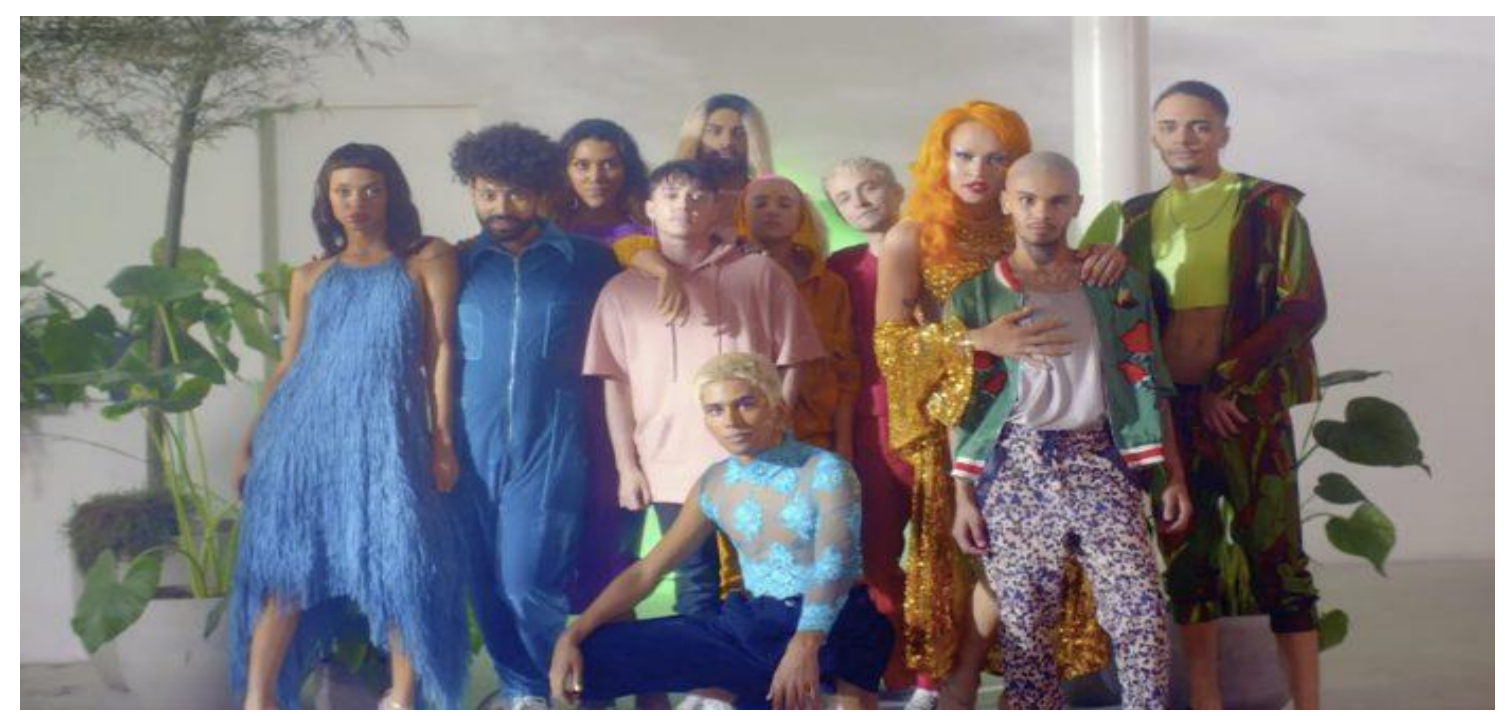

Figura 2. Personajes posan para la foto al final de la campaña

Fuente: vídeo de la campaña transmitida por la agencia AlmapBBDO (2019). 
Como la figura retrata bien, la pieza publicitaria en cuestión fue enfática en comprender que el ser humano debería ser el protagonista de la campaña, que enfatiza el espíritu humano del trabajo de Kotler et al. (2019), al tratar de contribuir a la resolución de problemas sociales a través de las preocupaciones y necesidades manifestadas por las propias demandas de la sociedad, especialmente las minorías.

El escenario es minimalista, cuyo objetivo es atraer la atención a los personajes, actores sociales reales y protagonistas de la campaña. El proceso de creación de la campaña generó la participación de actores sociales como una forma de representación, de acuerdo con la percepción de los sujetos.

En este sentido, todos los encuestados respaldan que los personajes presentes en la pieza publicitaria representan el movimiento LGBTQIA+, aunque no se explican todos sus componentes, lo que dirige la campaña a un supuesto estereotipo. Es posible observar las declaraciones de algunos de los sujetos cuestionados sobre la validez de la representación del movimiento bajo análisis en los personajes presentados por la campaña en la pregunta "¿Los personajes presentes en la campaña corresponden al Movimiento LGBTQIA+?": "Sí. Sin embargo, algunas son caricaturas. Creo que podrían explorar más la comunidad" (S1).

Los discursos de los entrevistados son unánimes con respecto a la creencia de que la mayor visibilidad de los actores sociales titulados LGBTIA+ (Rodrigues, 2018; Rodrigues y Carvalho, 2015) es beneficiosa para este movimiento, aunque la conexión fiel con la realidad de estos sigue siendo muy incipiente. Por eso, también difieren en cuanto al nivel considerado suficiente de presencia mediática necesaria para que se realicen debates sociales en el público en general sobre el tema.

En este sentido, el siguiente participante declara que se beneficia de la campaña publicitaria en cuestión.

Si!!! Es importante señalar que en el entorno LGBT aquellos que tienen más visibilidad son hombres que se identifican a sí mismos como homosexuales, por lo que siempre es importante demostrar que la clase LGBT no solo está formada por hombres homosexuales, sino por innumerables otras minorías, como: bisexuales, mujeres lesbianas, transexuales, queer y etc ... Y el video muestra este tipo de inclusión (S2).

El participante, declarado queer, destaca en su discurso la aclaración de un tema importante dentro de las discusiones sobre el movimiento de pertenencia de las identidades en cuestión. Sostiene que dentro de la comunidad LGBTQIA+ en sí hay minorías desatendidas por los medios de comunicación, como las clases híbridas, como los homosexuales y los bisexuales, y la presencia femenina. Luego, la visibilidad de estas clases en la pieza publicitaria puntúa, revelando el desarrollo de la publicidad en encuestas sociales que abarcan una mayor fidelidad a los preceptos del movimiento.

Los propios representantes del movimiento mostraron un nivel significativo de criticidad con respecto a sus percepciones de la campaña de la marca Doritos bajo análisis. Aunque da voz y visibilidad a la causa que defienden, la campaña tiene varias lagunas que, en opinión de los 
representantes, realizan esfuerzos incompletos para ayudar a que la causa alcance niveles más altos de aceptabilidad y comprensión.

Se observa, con un análisis más profundo de la pieza publicitaria en evidencia, que la intención es atraer a los consumidores a la marca, a través de la identificación con el concepto que ha estado proponiendo para ser una institución socialmente responsable y enfocada en resolver problemas. social, y aún presentando acciones estratégicas en las que vincula su imagen a la contribución de recursos financieros, obtenidos a través de la ganancia de los productos vendidos, a instituciones de apoyo a la comunidad de las cuales son miembros LGBTQIA+, simultáneamente pertenecientes a la causa.

\subsubsection{Análisis del mensaje de la campaña a partir de las percepciones presentes en los discursos de los sujetos}

Las entrevistas mostraron que las percepciones de los LGBTQIA+ consultados sobre la campaña, más específicamente la propuesta contenida en su logotipo "Nada es más bold que ser tú mismo", varían, pasando por las acciones de los sujetos sugeridas por verbos como sentir, respetar, ser, expresar e incluso alentar. Es el proceso de identificación con la idea central de la campaña, lo que explica el carácter de identificación que los sujetos deben tener para validar la comunicación utilizada.

Según uno de los entrevistados:

Hoy en día ser quién eres por completo (ideales, forma de comportarse, expresar voluntades, etc.) es principalmente un acto de coraje, por lo que la frase "Nada es más bold que ser tú mismo" significa exactamente eso: que hoy, no hay nada más atrevido que identificar, expresar y compartir quién eres (S3).

Hay un atractivo emocional, a veces exigido por el público objetivo, presente en este tipo de campaña. Existe la creencia unánime de que la presencia en los medios de este tipo de discusión, en particular las publicaciones que favorecen la difusión de una visión esclarecedora sobre la causa LGBTQIA+, es importante y debe mejorarse con frecuencia. Sin embargo, hacer que esta audiencia se vea en su plenitud es una tarea compleja y que requiere atención, como está presente en el discurso del sujeto 4 :

Creo que busca representar a la comunidad. Este tema es muy controvertido porque cada día la población LGBT tiene más representación y fuerza, por lo que es cada vez más común que se dirija algo o que tenga elementos que se parezcan a esta comunidad. Sin embargo, como se mencionó anteriormente, identifiqué elementos que promueven la inclusión (S4).

En cuanto a la cuestión del objetivo intrínseco más importante que la marca pretende alcanzar (Bragaglia, 2019; Gastaldo, 2013), los sujetos coinciden en que la campaña busca representar a la comunidad LGBTQIA+ y que todo el proceso de información va más allá de una simple pieza publicitaria para publicitar un producto o una marca, ya que también Puede anunciar una idea. Aquí están las percepciones de algunos encuestados sobre la intención social real de campañas como esta: "Yo creo que sí. Va más allá del marketing, unir la marca y asignar lucros a las instituciones es un ejemplo de esto" (S5). 
El sujeto 5 (S5) se refiere a la donación de parte de los recursos financieros recaudados por la campaña a instituciones que apoyan la causa LGBTQIA+. En su opinión, la pieza publicitaria refleja que la empresa creativa tenía una actitud que va más allá del marketing y dirige sus esfuerzos para ayudar a la causa social.

Del mismo modo, esta comprensión se puede profundizar al reflexionar sobre la posibilidad de hibridación en la estrategia publicitaria con un enfoque social (Kotler et al., 2019): "Los dos. Tanto para mostrar un poco de la comunidad LGBT como para publicitar su publicidad" (S6).

Estos dos discursos aclaran dos puntos importantes: la presencia significativa de inclusión en la campaña y la estrategia que las marcas están estudiando y ejecutando en función de la representatividad y la fuerza de la causa social en discusión. Va más allá de una simple discusión de buenas intenciones, pero se centra en analizar si estas estrategias publicitarias aportan beneficios a la sociedad, lo que ya justificaría su permanencia e intensificación.

A pesar de esto, cuando se trataba de los personajes elegidos para representar la causa social considerada, con respecto a inspirar a las personas a comprender qué es el movimiento LGBTQIA+, las opiniones diferían. Se enfatiza la crítica que algunos sujetos exponen al criticar la restricción del Movimiento LGBTQIA+ a algunos componentes y no a la exploración de todo el universo de personas: leen actores sociales diferentes de los presentados, que también son parte y tienen un sentido de pertenencia, aunque se consideran diferentes. de los estilos presentados por la campaña.

Es unánime entre los temas que la campaña en cuestión, aunque dotada de buena intención, necesita madurar socialmente para que realmente llegue al corazón del problema social con respecto a los actores sociales que representa, evidenciado en el siguiente discurso del sujeto 7: "Pondría un poco más de lucha y de los sufrimientos que sufre la clase LGBT, desafortunadamente esta violencia que sufrimos puede ser un elemento más llamativo para los anuncios, ya que es visual e impactante" (S7).

El tono obstinado del sujeto refleja su percepción sobre las luchas, aquí entendidas como las dificultades vivas de estas personas, de los miembros de los movimientos LGBTQIA+, quienes, con dificultades en la participación social, necesitan espacio en los medios y, en consecuencia, una mayor comprensión de la sociedad sobre sus derechos (Facchini, 2009; Ferreira y Sacramento, 2019; Rodrigues y Carvalho, 2015).

\section{CONCLUSIONES}

Se concluye que este artículo buscó hacer una interpretación pluralista de las percepciones de los sujetos entrevistados pertenecientes a la comunidad LGBTQIA+ sobre la campaña Doritos Rainbow del año 2019, a fin de encontrar oportunidades para optimizar la actividad publicitaria, a través de la producción social, como la visibilidad LGBTQIA+ en publicidad brasileña. Para eso, tenía percepciones variadas y apoyadas por la literatura.

La información obtenida en la literatura fue importante para la dirección de la investigación, ya que proporcionó la interpretación necesaria para los deseos de los sujetos involucrados y de los 
conceptos existentes en los temas abordados. $\mathrm{Y}$, al mismo tiempo, proporcionaron un avance teórico, aunque preliminar, sobre los estudios que dilucidan las discusiones al respecto.

Los resultados obtenidos revelaron el público objetivo de la campaña, el concepto del mensaje, los objetivos de la marca, para que pudieran hacer interpretaciones asertivas desde la perspectiva de los sujetos LGBTQIA+ y sus expectativas. Por un lado, existe una opinión común sobre la necesidad de centrar las campañas publicitarias en promover la visibilidad de la causa en estudio, lo que de hecho puede lograr la campaña publicitaria analizada. Por otro lado, existe una crítica de que las campañas deben estudiar más los signos, las características, filosofía popular y la historia de las causas sociales adheridas a fin de enfocar y difundir mejor información creíble, lo que respalda una de las hipótesis planteadas por este trabajo.

Las posibles limitaciones de este estudio pueden considerarse la característica transversal de la investigación en lugar de un enfoque longitudinal, los sujetos entrevistados se concentran en un lugar (por criterios de accesibilidad) y las percepciones sobre el tema central, la visibilidad del Movimiento LGBTQIA+ en la publicidad brasileña, aportar ideas de una sola campaña. Sin embargo, para el estudio propuesto, esta delimitación aseguró buenos resultados y discusiones significativas y contribuyentes para el desarrollo del conocimiento sobre los temas explorados.

Como más significativo, se concluye que las percepciones de los sujetos enfatizan que, en este campo de discusión, es necesario considerar, al optar por usar una causa social compleja, varios factores como la historia de esta causa, las percepciones de los sujetos que son parte a partir de ello, la deconstrucción de paradigmas erróneos y una mejora en la representatividad de los miembros con especial atención.

Por lo tanto, se argumenta que puntos como la aceptabilidad de las campañas por parte de los miembros del movimiento en comparación con el resto de la sociedad, los métodos para producir campañas publicitarias para LGBTQIA+ y las barreras y facilitadores para estas campañas pueden considerarse en futuras investigaciones.

\section{Referencias}

Bortolozzi, R. (2019). Mosaico de Purpurina: revisitando a História do Movimento LGBT no Brasil. Revista Eletrônica de Comunicação, Informação e Inovação Em Saúde, 13(3). doi: https://doi.org/10.29397/reciis.v13i3.1831

Bragaglia, A. P. (2019). A apropriação do social pela publicidade. Signos Do Consumo, 11(1), 84-96. doi: https://doi.org/10.11606/issn.1984-5057.v11i1p84-96

Carvalho, L., \& Lisboa Filho, F. F. (2019). Representações LGBTQIA+ e estudos culturais: invisibilidades da diversidade de gênero em audiovisuais publicitários de moda. Revista Eletrônica de Comunicação, Informação e Inovação Em Saúde, 13(3). doi: https://doi.org/10.29397/reciis.v13i3.1726

Duarte, J., \& Barros, A. (2006). Métodos e técnicas de Pesquisa em Comunicação. $2^{a}$ edição. São Paulo: Atlas. Disponible en: <http://www.revistas.usp.br/organicom/article/download/138920/134268/>. Acceso en: 20 julio 2020. 
Facchini, R. (2009). Entre compassos e descompassos: um olhar para o" campo" e para a" arena" do movimento LGBT brasileiro. Bagoas-Estudos Gays: Gêneros e Sexualidades, 3(04). Disponible en: < https://periodicos.ufrn.br/bagoas/article/view/2300/0>. Acceso en: 20 julio 2020.

Facchini, R., Rodrigues, J. (2018). É preciso estar atento (a) e forte: histórico do movimento LGBT e a conjuntura atual. In L. Nogueira, E. Hilário, T. T. Paz, \& K. Marro (Eds.), Hasteemos a bandeira colorida: diversidade sexual e de gênero no Brasil (pp. 231-262). São Paulo: Expressão Popular.

Ferreira, V., \& Sacramento, I. (2019). Movimento LGBT no Brasil: violências, memórias e lutas. Revista Eletrônica de Comunicação, Informação e Inovação Em Saúde, 13(2). doi: https://doi.org/10.29397/reciis.v13i2.1826

Gastaldo, E. (2013). Publicidade e Sociedade: uma perspectiva antropológica. Editoria Sulina, Editora Meridional Ltda.

Gregolin, M. D. R. V. (1995). A análise do discurso: conceitos e aplicações. ALFA: Revista de Linguística. Disponible en: <https://periodicos.fclar.unesp.br/alfa/article/view/3967>. Acceso en: 20 julio 2020.

Kotler, P., Kartajaya, H., \& Setiawan, I. (2010). Marketing 3.0: From products to customers to the human spirit. John Wiley \& Sons. Disponible en: < https://link.springer.com/chapter/10.1007/978-98110-7724-1 10>. Acceso en: 20 julio 2020.

Kotler, P., \& Keller, K. L. (2012). Administração de Marketing. Tradução de Sônia Midori Yamamoto. São Paulo: Pearson Education do Brasil.

Llewellyn, S., \& Northcott, D. (2007). The" singular view" in management case studies. Qualitative Research in Organizations and Management, 2(3), 194. doi: https://doi.org/10.1108/17465640710835355

Malhotra, N. K. (2011). Pesquisa de marketing: foco na decisão. São Paulo: Pearson Prentice Hall.

Minayo, M. C. (2017). Amostragem e saturação em pesquisa qualitativa: consensos e controvérsias. Revista Pesquisa Qualitativa, 5(7), 1-12. Disponible en: < https://editora.sepq.org.br/index.php/rpq/article/view/82/59>. Acceso en: 20 julio 2020.

Patton, M. Q. (2014). Qualitative research \& evaluation methods: Integrating theory and practice. Sage publications. Disponible en: <https://us.sagepub.com/en-us/nam/qualitative-researchevaluation-methods/book232962>. Acceso en: 21 julio 2020.

Pêcheux, M. (1990). O discurso. Estrutura Ou Acontecimento. Campinas: Pontes.

Peralta, L. M., Martins, K. M., \& da Silva Santana, P. (2019). Breve análise sobre o perfil do consumidor homoafetivo/Brief analysis on the homoaffective consumer profile. Brazilian Journal of Development, 5(7), 8629-8650. doi: https://doi.org/10.34117/bjdv5n7-076

Rocha, D., \& Deusdará, B. (2005). Análise de Conteúdo e Análise do Discurso: aproximações e afastamentos na (re) construção de uma trajetória. Alea: Estudos Neolatinos, 7(2), 305-322. doi: https://doi.org/10.1590/S1517-106X2005000200010

Rodrigues, A. I. (2018). Entre as representações e as repercussões das homossexualidades: uma análise da publicidade veiculada na TV aberta e seus desdobramentos na rede social. ConexãoComunicação e Cultura, 16(32). Disponible en: < http://www.ucs.br/etc/revistas/index.php/conexao/article/view/5087>. Acceso en: 21 julio 2020

Rodrigues, A. I., \& Carvalho, A. de. (2015). Desde a década de setenta, em setenta comerciais: as representações LGBT na publicidade e propaganda veiculadas na televisão brasileira. Encontro Nacional de História Da Mídia (10.: 2015 Jun. 3-5: Porto Alegre, RS). Anais. [Porto Alegre, RS: Alcar, 2015]. Disponible en: <http://hdl.handle.net/10183/132742>. Acceso en: 21 julio 2020. 
Santos, G. S., \& da Cunha, J. L. R. (2019). Representações sociais e narrativas publicitárias: orgulho de ser LGBT pela Skol. Dispositiva, 8(13), 43-65. doi: https://doi.org/10.5752/P.2237-9967.2019v8n13p43-65 